\title{
Multiple Matrix Rank Constrained Optimization for Optimal Power Flow over Large Scale Transmission Networks
}

\author{
Y. Shi ${ }^{1}$, H. D. Tuan ${ }^{1}$, S. W. Su ${ }^{1}$ and A. V. Savkin ${ }^{2}$ \\ ${ }^{1}$ Faculty of Engineering and Information Technology, University of Technology, Sydney, NSW 2007, Australia \\ ${ }^{2}$ School of Electrical Engineering and Telecommunications, University of New South Wales, Sydney, NSW 2052, Australia
}

\begin{abstract}
Keywords: Optimal Power Flow (OPF), Transmission Networks, Rank-one Matrix Constraint, Nonsmooth Optimization, Semi-Definite Programming (SDP).

Abstract: $\quad$ The optimal power flow (OPF) problem for power transmission networks is an NP-hard optimization problem with numerous quadratic equality and indefinite quadratic inequality constraints on bus voltages. The existing nonlinear solvers often fail in yielding a feasible solution. In this paper, we follow our previously developed nonsmooth optimization approach to address this difficult large-scale OPF problem, which is an iterative process to generate a sequence of improved solutions that converge to an optimal solution. Each iteration calls an SDP of a moderate dimension. Intensive simulations for OPF over networks with a large number of buses are provided to demonstrate the efficiency of our approach.
\end{abstract}

\section{INTRODUCTION}

The brain of a smart grid is advanced distribution management system (DMS), which is responsible for supervisory control and data acquisition in reactive dispatch, voltage regulation, contingency analysis, capability maximization and other smart operations. Optimal power flow (OPF), which determines a steady state operating point such that the cost of electric power generation is minimized under operating constraints, lies at the heart of DMS ((Carpentier, 1962; Huneault and Galiana, 1991; Momoh et al., 1999; Pandya and Joshi, 2008) and references therein). The OPF problem is typically nonlinear and nonconvex due to the multiple quadratic equality and indefinite quadratic inequality constraints on the voltages variables in expressing the bus interconnections, hardware operating capacity and balance between power demand and supply. These nonlinear constraints are difficult so the state-of-the-art nonlinear optimization solvers may converge to just stationary points (see (Bukhsh et al., 2013) and references therein), which are even not necessarily feasible.

There has been a renewed attention on the application of semi-definite programming (SDP) to the OPF problem. As a nonconvex quadratic optimization, OPF can be easily recast by convex quadratic optimization with the additional nonconvex rank-one constraint on the matrix $W=V V^{H}$ of the outer prod- uct of voltage vector variable $V$ (Bai et al., 2008; Lavaei and Low, 2012). The matrix solution of the semi-definite relaxation (SDR) by dropping the rankone matrix constraint, is of rank-one and therefore provides the global OPF solution in the power distribution networks (Madani et al., 2015b) or in few modified IEEE networks (Lavaei and Low, 2012). Often, a low-rank matrix solution of SDR is of not rank-one and cannot result in a feasible point of the original nonconvex OPF problem (Lavaei and Low, 2012; Madani et al., 2015b; Madani et al., 2015a). An another setback of using the such matrix variable $W \in \mathbb{C}^{n \times n}$ for a network of $n$ buses is that its dimension $n(n+1) / 2$ increases dramatically with respect to $n$. For instance, for moderate $n=150$ and $n=300$ such $W$ is equivalent to $150 \times 151 / 2=11.325$ and $300 \times 301 / 2=45.150$ complex scalar variables. On the other hand, all large scale networks are sparse in the sense that the number of the flow lines connecting buses is relatively moderate. This means that only a small portion of the crossed nonlinear terms $V_{k} V_{m}^{*}$ appearing in the constraints. References (Molzahn et al., 2013; Andersen et al., 2014; Madani et al., 2015a) suggest to formulate OPF as optimization in multiple matrix variables of outer products of overlapped subsets of voltages. Obviously, it is hardly expected that SDR by dropping the rank-one constraints on all these multiple outer products would have all rank-one solution. Consequently, the matrix solution of SDR does 
not have any physical meaning.

To address the rank-one issue, in (Shi et al., 2015), we have proposed a rank-reduced optimization to address the rank-one for the matrix variable $W$ of the total voltages, which works very well and practically for moderate networks up to 300 buses. In this paper, we will further develop this rank-reduced optimization technique for its large-scale OPFs up to a few thousands buses.

The paper is structured as follows. Section 2 is devoted to the problem formulation, its challenges and its computational solution development. Section 3 provides simulation to show the efficiency of our methods. The conclusions are drawn in Section 4.

The notation used in the paper is standard. More specifically, $j$ denotes the imaginary unit, $M \succeq 0$ means that the Hermitian symmetric matrix $M$ is positive semi-definite, $\operatorname{rank}(M)$ is the rank of the matrix $M ; \mathfrak{R}(\cdot)$ and $\mathfrak{I}(\cdot)$ denote the real and imaginary parts of a complex quantity. $a \leq b$ for two complex numbers $a$ and $b$ is componentwise understood, i.e. $\mathfrak{R}(a) \leq \mathfrak{R}(b)$ and $\mathfrak{I}(a) \leq \mathfrak{I}(b)$. $\langle.,$.$\rangle is the dot prod-$ uct of matrices, while $\left\{A_{i}\right\}_{i=1, \ldots, n}$ denotes the matrix with diagonal blocks $A_{i}$ and zero off-diagonal blocks.

\section{LARGE-SCALE OPTIMAL POWER FLOW PROBLEM AND CHALLENGES}

Consider an AC electricity transmission network with a set of buses $\mathcal{N}:=\{1,2, \cdots, n\}$. The buses are connected through a set of flow lines $\mathcal{L} \subseteq \mathcal{N} \times \mathcal{N}$, i.e. bus $m$ is connected to bus $k$ if and only if $(m, k) \in \mathcal{L}$. Accordingly, $\mathcal{N}(k):=\{m \in \mathcal{N}:(m, k) \in \mathcal{L}\}$. The power demanded at bus $k \in \mathcal{N}$ is

$$
S_{L_{k}}=P_{L_{k}}+j Q_{L_{k}},
$$

where $P_{L_{k}}$ and $Q_{L_{k}}$ are the real and reactive power. A subset $\mathcal{G} \subseteq \mathcal{N}$ of buses is supposed to be connected to generators. Any bus $k \in \mathcal{N} \backslash \mathcal{G}$ is thus not connected to generators. Other notations are: $(i)$ $Y=\left[y_{k m}\right]_{(k, m) \in \mathcal{N} \times \mathcal{N}} \in \mathbb{C}^{n \times n}$ is the admittance matrix (Zimmerman et al., 2011). Each $y_{k m}$ is the mutual admittance between bus $k$ and bus $m$, so $y_{k m}=y_{m k}$ $\forall(k, m) \in \mathcal{L}$; (ii) $V$ is the complex voltage vector, $V=$ $\left[V_{1}, V_{2}, \cdots, V_{n}\right]^{T} \in \mathbb{C}^{n}$, where $V_{k}$ is the complex voltage injected to bus $k \in \mathcal{N}$; (iii) $I$ is the complex current vector, $I=Y V=\left[I_{1}, I_{2}, \cdots, I_{n}\right]^{T} \in \mathbb{C}^{n}$, where $I_{k}$ is the complex current injected to bus $k \in \mathcal{N} ;(i v) I_{k m}$ is the complex current in the power line $(k, m) \in \mathcal{L}$, $\sum_{m \in \mathcal{N}(k)} I_{k m}=I_{k}=\sum_{m \in \mathcal{N}(k)} y_{k m} V_{m} ;(v) S_{k m}=P_{k m}+j Q_{k m}$ is the complex power transferred from bus $k$ to bus $m$, where $P_{k m}$ and $Q_{k m}$ represent the real and reactive transferred power; ( $v i) S_{G_{k}}=P_{G_{k}}+j Q_{G_{k}}$ is the complex power injected by bus $k \in \mathcal{G}$, where $P_{G_{k}}$ and $Q_{G_{k}}$ represent the real and reactive generated power.

For each bus $k$, it is obvious that $S_{G_{k}}-S_{L_{k}}=$ $\left(P_{G_{k}}-P_{L_{k}}\right)+j\left(Q_{G_{k}}-Q_{L_{k}}\right)=V_{k} \sum_{m \in \mathcal{N}(k)} V_{m}^{*} y_{k m}^{*}$. Therefore, we can express the real generated power $P_{G_{k}}$ and reactive generated power $Q_{G_{k}}$ at bus $k$ as $P_{G_{k}}=P_{L_{k}}+\mathfrak{R}\left(\sum_{m \in \mathcal{N}(k)} V_{k} V_{m}^{*} y_{k m}^{*}\right)$ and $Q_{G_{k}}=Q_{L_{k}}+$ $\mathfrak{I}\left(\sum_{m \in \mathfrak{N}(k)} V_{k} V_{m}^{*} y_{k m}^{*}\right)$.

The objective of OPF is to minimize the following cost function of real active generated power $P_{G}: f\left(P_{G}\right)=\sum_{k \in \mathcal{G}}\left(c_{k 2} P_{G_{k}}^{2}+c_{k 1} P_{G_{k}}+c_{k 0}\right)$ with $c_{k 2}>$ $0, c_{k 1}, c_{k 0}$ given, which is a function of the bust voltages $V$ :

$$
\begin{aligned}
f(V)= & \sum_{k \in \mathcal{G}}\left[c_{k 2}\left(P_{L_{k}}+\mathfrak{R}\left(\sum_{m \in \mathcal{N}(k)} V_{k} V_{m}^{*} y_{k m}^{*}\right)\right)^{2}\right. \\
+ & \left.c_{k 1}\left(P_{L_{k}}+\mathfrak{R}\left(\sum_{m \in \mathcal{N}(k)} V_{k} V_{m}^{*} y_{k m}^{*}\right)\right)+c_{k 0}\right] .(1)
\end{aligned}
$$

Accordingly, the following OPF problem is formulated

$$
\min _{V \in \mathbb{C}^{n}} f(V) \quad \text { s.t. (2a) }
$$

$-P_{L_{k}}-j Q_{L_{k}}=\sum V_{k} V_{m}^{*} y_{k m}^{*}, k \in \mathcal{N} \backslash \mathcal{G},(2 \mathrm{~b})$

$$
\begin{array}{r}
P_{G_{k}}^{\min } \leq P_{L_{k}}+\Re\left(\sum_{m \in \mathcal{N}(k)} V_{k} V_{m}^{*} y_{k m}^{*}\right) \leq P_{G_{k}}^{\max }, k \in \mathcal{G}, \\
Q_{G_{k}}^{\min } \leq Q_{L_{k}}+\mathfrak{I}\left(\sum_{m \in \mathcal{N}(k)} V_{k} V_{m}^{*} y_{k m}^{*}\right) \leq Q_{G_{k}}^{\max }, k \in \mathcal{G}, \\
V_{k}^{\min } \leq\left|V_{k}\right| \leq V_{k}^{\max }, k \in \mathcal{N}, \\
\left|S_{k m}\right|=\left|V_{k} V_{m}^{*} y_{k m}^{*}\right| \leq S_{k m}^{\max }, \forall(k, m) \in \mathcal{L} \\
\left|V_{k}-V_{m}\right| \leq V_{k m}^{\max },(k, m) \in \mathcal{L}, \\
\left|\arg \left(V_{k}\right)-\arg \left(V_{m}\right)\right| \leq \theta_{k m}^{\max },(k, m) \in \mathcal{L},
\end{array}
$$

where $(2 b)$ is the equation of the balance between the demand and supply power at bus $k \in \mathcal{N} \backslash \mathcal{G}$, (2c)(2d) are the power generation bounds, where $P_{G_{k}}^{\text {min }}$, $Q_{G_{k}}^{\min }$ and $P_{G_{k}}^{\max }, Q_{G_{k}}^{\max }$ are the lower bound and upper bound of the real power reactive power generations, respectively, (2e) are the voltage amplitude bounds, (2f)-(2h) are capacity limitations, where the line currents between the connected buses are constrained by $(2 \mathrm{f})$, while $(2 \mathrm{~g})-(2 \mathrm{~h})$ guarantee the voltage balance in terms of their magnitude and phases (Zimmerman et al., 2011).

Introducing $W_{k m}=V_{k} V_{m}^{*}, k=1, \ldots, n ; m=1, \ldots, n$ and $W=\left[W_{k m}\right]_{k, m=1, \ldots, n}$, the problem (2) is recast to 
the following optimization problem

$$
\begin{array}{r}
\min _{W \succeq 0} F(W) \\
-P_{L_{k}}-j Q_{L_{k}}=\sum_{m \in \mathcal{N}(k)} W_{k m} y_{k m}^{*} k \in \mathcal{N} \backslash \mathcal{G}, \\
P_{G_{k}}^{\min } \leq P_{L_{k}}+\mathfrak{R}\left(\sum_{m \in \mathcal{N}(k)} W_{k m} y_{k m}^{*}\right) \leq P_{G_{k}}^{\max }, k \in \mathcal{G}, \\
Q_{G_{k}}^{\min } \leq Q_{L_{k}}+\mathfrak{I}\left(\sum_{m \in \mathcal{N}(k)} W_{k m} y_{k m}^{*}\right) \leq Q_{G_{k}}^{\max }, k \in \mathcal{G}, \\
\left(V_{k}^{\min }\right)^{2} \leq W_{k k} \leq\left(V_{k}^{\max }\right)^{2}, k \in \mathcal{N}, \\
\left|W_{k m} y_{k m}^{*}\right| \leq S_{k m}^{\max },(k, m) \in \mathcal{L}, \\
W_{k k}+W_{m m}-W_{k m}-W_{m k} \leq\left(V_{k m}^{\max }\right)^{2},(k, m) \in \mathcal{L}, \\
\mathfrak{I}\left(W_{k m}\right) \leq \mathfrak{R}\left(W_{k m}\right) \tan \theta_{k m}^{\max },(k, m) \in \mathcal{L}, \\
\operatorname{rank}(W)=1,
\end{array}
$$

where $F(W)=\sum_{k \in \mathcal{G}}\left[c_{k 2}\left(P_{L_{k}}+\Re\left(\sum_{m \in \mathcal{N}(k)} W_{k m} y_{k m}^{*}\right)\right)^{2}+\right.$ $\left.c_{k 1}\left(P_{L_{k}}+\mathfrak{R}\left(\sum_{m \in \mathcal{N}(k)} W_{k m} y_{k m}^{*}\right)\right)+c_{k 0}\right]$, which is convex quadratic in $W_{k m}$.

SDR approach (see e.g. (Lavaei and Low, 2012)) is to drop the difficult rank-one constraint (3i) for SDR. If the solution of such relaxed SDP is of rank-one, i.e. it satisfies the nonconvex rank-one constraint (3i) then it obviously leads to the global solution of the nonconvex optimization problem (3). Otherwise even a feasible solution of (3) is hardly obtained from a solution of this SDR.

In (Shi et al., 2015), we suggest to address (3) by the following spectral optimization

$$
\begin{array}{r}
\min _{W \succeq 0} F_{\mu}(W):=F(W)+\mu(\operatorname{Trace}(W) \\
\left.-\lambda_{\max }(W)\right) \quad \text { s.t. }(3 b)-(3 h),
\end{array}
$$

where $\lambda_{\max }(W)$ stands for the maximal eigenvalue of $W$ and $\mu$ is a penalty parameter. Note that $\operatorname{Trace}(W)-\lambda_{\max }(W)=0$. The nonnegative quantity $\operatorname{Trace}(W)-\lambda_{\max }(W)$ can therefore be used to measure the degree of satisfaction of the matrix rank-one constraint (3i). Without square on the factor of $\mu$, the penalization $\operatorname{Trace}(W)-\lambda_{\max }(W)$ in (4) is exact, meaning that the constraint $\operatorname{Trace}(W)=\lambda_{\max }(W)$ can be satisfied by a minimizer of (4) with a finite value of $\mu$ (see e.g. (Bonnans et al., 2006, Chapter 16)). This is generally considered as a sufficiently nice property to make such exact penalization attractive.

Function $\lambda_{\max }(W)$ is nonsmooth but is lower bounded by $\lambda_{\max }(W)=\max _{\|w\|=1} w^{H} W w \geq$ $\left(w_{\max }^{(\mathrm{\kappa})}\right)^{H} W w_{\max }^{(\mathrm{\kappa})}$, where $w_{\max }^{(\mathrm{\kappa})}$ is the normalized eigenvector corresponding to the eigenvalue $\lambda_{\max }\left(W^{(\kappa)}\right)$, i.e. $\lambda_{\max }\left(W^{(\kappa)}\right)=\left(w_{\max }^{(\kappa)}\right)^{H} W^{(\kappa)} w_{\max }^{(\kappa)}$.

Therefore, for any $W^{(\kappa)}$ feasible to the convex constraints (3b)-(3h), the following convex optimization problem provides an upper bound for the nonconvex optimization problem (4)

$$
\begin{aligned}
& \min _{W \succeq 0} F^{(\kappa)}(W):=F(W)+\mu[\operatorname{Trace}(W) \\
& \left.-\left(w_{\max }^{(\kappa)}\right)^{H} W w_{\max }^{(\kappa)}\right] \quad \text { s.t. } \quad(3 b)-(3 h)
\end{aligned}
$$

because $F^{(\kappa)}(W) \geq F_{\mu}(W) \quad \forall W \succeq 0$.

Suppose that $W^{(\kappa+1)}$ is the optimal solution of SDP (5). Since $W^{(\kappa)}$ is also feasible to (5) with $F_{\mu}\left(W^{(\kappa)}\right)=F^{(\kappa)}\left(W^{(\kappa)}\right)$, it is true that $F_{\mu}\left(W^{(\kappa)}\right)=$ $F^{(\kappa)}\left(W^{(\kappa)}\right) \geq F^{(\kappa)}\left(W^{(\kappa+1)}\right) \geq F_{\mu}\left(W^{(\kappa+1)}\right)$, so $W^{(\kappa+1)}$ is a better feasible point of (4) than $W^{(\kappa)}$.

The common drawback of using the structurefree matrix variable $W \in \mathbb{C}^{n \times n}$ is that its dimension $n(n+1) / 2$ increases dramatically with respect to the number $n$ of the network buses. For instance, for $n=150$ and $n=300$ such $W$ is equivalent to $150 \times$ $151 / 2=11.325$ and $300 \times 301 / 2=45.150$ complex scalar variables. On the other hand, most large scale networks are sparse in the sense that the number of the flow lines connecting buses is relatively moderate. There is only a small portion of the crossed terms $V_{k} V_{m}^{*}$ appearing in the nonlinear constraints (2b)-(2h) so such matrix variable $W$ contains many redundant $V_{k} V_{m}^{*}$. References (Molzahn et al., 2013; Andersen et al., 2014; Madani et al., 2015a) decompose the set $\mathcal{N}:=\{1,2, \ldots, n\}$ into $I$ possibly overlapped subset $\mathcal{N}_{i}=\left\{i_{1}, \ldots, i_{N_{i}}\right\}$ called by bags, such that for each $i=1,2, \ldots, I$

$$
i_{\ell} \in \mathcal{N}\left(i_{\ell+1}\right), \ell=1, \ldots, N_{i}-1 \quad \text { and } \quad i_{N_{i}} \in \mathcal{N}\left(i_{1}\right) .
$$

The set of bags can be reset such that its bags are of relatively same size.

Define the Hermitian symmetric matrix variables

$$
W^{i}=\left[W_{i_{k} i_{m}}\right]_{k, m=1, . ., N_{i}} \in \mathbb{C}^{N_{i} \times N_{i}}, i=1,2, \ldots, I
$$

and $W=\operatorname{diag}\left\{W^{i}\right\}_{i=1, \ldots, I}$. By replacing $W_{k m}=V_{k} V_{m}^{*}$ in (1) and constraints (2b)-(2h), we have the following equivalent optimization reformulation for (2)

$\min _{W \succeq 0} F(W) \quad$ s.t. $(3 b)-(3 h), \operatorname{rank}\left(W^{i}\right)=1, i=1, \ldots, I$.

Reference (Molzahn et al., 2013; Andersen et al., 2014) consider (2) just by dropping all rank-one constraints in (7), while (Madani et al., 2015a) used a penalized SDR for locating a low-rank semi-definite solution $W^{i}$. In the end, a rank-one solution could not be found.

Now, we propose the following algorithm for computational solution of (7). 
Large-scale Nonsmooth Optimization Algorithm (NOA).

Initialization. Solve SDP

$$
\min _{W} F(W) \quad \text { s.t. } \quad(3 b)-(3 h)
$$

to find its solution $W^{(0)}:=\operatorname{diag}\left\{W^{i,(0)}\right\}_{i=1, \ldots, I}$. If $\operatorname{rank}\left(W^{i,(0)}\right) \equiv 1$ stop: $W^{(0)}$ is the global solution of the nonconvex optimization problem (7). Otherwise define $\mathcal{L}^{(0)}=\left\{i \in\{1, \ldots, I\}: \operatorname{rank}\left(W^{i,(0)}\right)=1\right\}$

Step 1. For $\kappa=0,1, .$. , and the normalized eigenvector $w_{\max }^{i,(\kappa)}$ corresponding to the eigenvalue $\lambda_{\max }\left(W^{i,(\kappa)}\right)$ of $W^{i,(\kappa)}$ solve

$$
\begin{array}{r}
\min _{W} F^{(\kappa)}(W):=F(W)+\mu \sum_{i \notin \mathcal{L}(\kappa)}\left[\operatorname{Trace}\left(W^{i}\right)\right. \\
\left.-\left(w_{\max }^{i,(\kappa)}\right)^{H} W^{i} w_{\max }^{i,(\kappa)}\right] \quad \text { s.t. } \quad(3 b)-(3 h), \\
\operatorname{Trace}\left(W^{i}\right)-\left(w_{\max }^{i,(\kappa)}\right)^{H} W^{i} w_{\max }^{i,(\kappa)} \leq \varepsilon_{t o l}, i \in \mathcal{L}^{(\kappa)}
\end{array}
$$

to find its solution $W^{(\kappa+1)}:=\operatorname{diag}\left\{W^{i,(\kappa+1)}\right\}_{i=1,2, \ldots, I}$. Stop if $\operatorname{rank}\left(W^{i,(\kappa+1)}\right)=1$ for all $i=1,2, \ldots ., I$. Otherwise define $\mathcal{L}^{(\kappa+1)}=\{i \in\{1, \ldots, I\}$ : $\left.\operatorname{rank}\left(W^{i,(\kappa+1)}\right)=1\right\}$ and reset $\kappa=\kappa+1$.

Remark. The constraint (9b) implies that $W^{i}$ must be a rank-one solution, so it is introduced to prevent good bags turning out to be bad.

Similarly to (Shi et al., 2015), the following proposition holds.

Proposition 1. Initialized by any feasible point $W^{i,(0)}$ of SDP (8), $\left\{W^{(\kappa)}\right\}$ is a sequence of improved feasible points of the nonconvex optimization problem

$$
\begin{array}{r}
\min _{W} F^{(\kappa)}(W):=F(W)+\mu \sum_{i}\left[\operatorname{Trace}\left(W^{i}\right)\right. \\
\left.-\lambda_{\max }\left(W^{i}\right)\right] \quad \text { s.t. } \quad(3 b)-(3 h),
\end{array}
$$

which converges to a point satisfying first-order necessary optimality conditions.

\section{SIMULATION RESULTS}

The hardware and software facilities for our computational implementation are:

- Processor: Intel(R) Core(TM) i5-3470 CPU @ 3.20GHz;

- Software: Matlab version R2013b;

- Matlab toolbox: Matpower version 5.1(Zimmerman et al., 2011) to compute the admittance matrix $Y$ from the power system data; CVX with SDPT3 solver for SDP (5).

\subsection{Polish-2383wp System}

Polish-2383wp system is part of the European UCTE system, with 2383 buses, 327 generators and 2896 transmission line, which lead to 2056 nonlinear constraints in (2b). Thus, there are $2383 \times 2384 / 2=$ 2840536 complex scalar variables in the $W$ matrix. However $W$ is sparse matrix, most element are 0 , thus we apply the Large-Scale NOA as follow,

Initialization. Following (Madani et al., 2015a) to decompose the 2383 buses into 2383 overlapped bags. The largest bag size is 24 , while the smallest bag size is 1. After the bags decomposition, the total number of the complex decision variables in (8) is 23199 vs 2840536 complex decision variables in (3). Solve (8) over the 2383 bags, we find that there are 653 bad bags, which means the rank of the 653 bags are more than 1 . The largest bad bag size is 24 , while the smallest bad bag size is 2 . Other 1720 good bags are all rank-1. Then go to next step.

Step 1. Set $\varepsilon_{\text {tol }}=10^{-6}$ and put the 1720 good bags in constraint (9b), solve (9), after 8 iterations, the bad bags number turns to be 13 , while the good bags number turns to be 2370 . Among the 13 bad bags, six have size 3 , four have size 4 , one has size 5 and two have size 6 .

Step 2. Put the 2370 good bags in constraint (9b), solve (9), after 5 iterations, the bad bags number turns to be 10, while the good bags number turns to be 2373. Among the 10 bad bags, six have size 3 , three have size 4 , and one has size 6 .

Step 3. Put the 2373 good bags in constraint (9b), solve (9), after 5 iterations, the bad bags number turns to be 5, while the good bags number turns to be 2378 . Among the 5 bad bags, two have size 3, two have size 4 , and one has size 6 .

Step 4. Put the 2378 good bags in constraint (9b), solve (9), after 5 iterations, the bad bags number turns to be 2, while the good bags number turns to be 2381 . Among the 2 bad bags, one is size 3 , the other is size 4.

Step 5. Put the 2381 good bags in constraint (9b), solve (9), after 5 iterations, the bad bags number turns to be 0 . The optimal objective value is $1.9464 \times 10^{6}$.

It should be noted that, in step 4 , two multiplier $\mu$ were added in the bags penalized term, to increase the convergence speed of bag-2011 and bag-2254, where $\mu=4$. In step 5 , bag-711 was multiplied by 4 as well.

\subsection{Polish-2736sp System}

Polish-2736sp system represents the Polish networks during summer 2004 peak conditions, with 2736 buses, 420 generators and 3504 transmission line, 
which lead to 2316 nonlinear constraints in (2b). Thus, there are $2736 \times 2737 / 2=3744216$ complex scalar variables in the $W$ matrix. We apply the LargeScale NOA as follow,

Initialization. Following (Madani et al., 2015a) to decompose the 2736 buses into 2736 overlapped bags. The largest bag size is 24 , while the smallest bag size is 1 . After the bags decomposition, the total number of the complex decision variables in (8) is 27298 vs 3744216 complex decision variables in (3). Solve (8) over the 2736 bags, we find that there is only one bad bag with size 4 , more specifically, the second largest eigenvalue is only $3.4 \times 10^{-5}$, which is closely to rank-1 tolerance criterion $10^{-5}$.

Step 1. Set $\varepsilon_{\text {tol }}=10^{-5}$, put the 2735 good bags in constraint (9b), solve (9), after 1 iterations, the bad bags number turns to be 0 . The optimal objective value is $1.3125 \times 10^{6}$.

\subsection{Polish-2737sop System}

Polish-2737sop system is the Polish networks during summer 2004 peak conditions, with 2737 buses, 399 generators and 3506 transmission line, which lead to 2338 nonlinear constraints in (2b). Thus, there are $2737 \times 2738 / 2=3746953$ complex scalar variables in the $W$ matrix. We apply the Large-Scale NOA as follow,

Initialization. Following (Madani et al., 2015a) to decompose the 2737 buses into 2737 overlapped bags. The largest bag size is 24 , while the smallest bag size is 1 . After the bags decomposition, the total number of the complex decision variables in (8) is 27034 vs 3746953 complex decision variables in (3). Solve (8) over the 2737 bags, we find that there are three bad bags, whose size are all 2.

Step 1. Set $\varepsilon_{t o l}=10^{-5}$, put the 2734 good bags in constraint (9b), solve (9), after 1 iterations, the bad bags number turns to be 0 . The optimal objective value is $7.8235 \times 10^{5}$.

\subsection{Polish-2746wop System}

Polish-2746wop system is the Polish networks during winter 2003-04 off-peak conditions, with 2746 buses, 514 generators and 3514 transmission line, which lead to 2232 nonlinear constraints in (2b). Thus, there are $2746 \times 2747 / 2=3771631$ complex scalar variables in the $W$ matrix. We apply the Large-Scale NOA as follow,

Initialization. Following (Madani et al., 2015a) to decompose the 2746 buses into 2746 overlapped bags. The largest bag size is 24 , while the smallest bag size is 1 . After the bags decomposition, the total num- ber of the complex decision variables in (8) is 29024 vs 3771631 complex decision variables in (3). Solve (8) over the 2746 bags, we find that there is only one bad bag with size 2 .

Step 1. Set $\varepsilon_{\text {tol }}=10^{-5}$, put the 2746 good bags in constraint (9b), solve (9), after 1 iterations, the bad bags number turns to be 0 . The optimal objective value is $1.2084 \times 10^{6}$.

\subsection{Polish-2746wp System}

Polish-2746wop system is the Polish networks during winter 2003-04 evening peak conditions, with 2746 buses, 520 generators and 3514 transmission line, which lead to 2226 nonlinear constraints in (2b). Thus, there are $2746 \times 2747 / 2=3771631$ complex scalar variables in the $W$ matrix. We apply the LargeScale NOA as follow,

Initialization. Following (Madani et al., 2015a) to decompose the 2746 buses into 2746 overlapped bags. The largest bag size is 24 , while the smallest bag size is 1 . After the bags decomposition, the total number of the complex decision variables in (8) is 28257 vs 3771631 complex decision variables in (3). Solve (8) over the 2746 bags, we find that there is only one bad bag with size 2 .

Step 1. Set $\varepsilon_{t o l}=10^{-5}$, put the 2746 good bags in constraint (9b), solve (9), after 1 iterations, the bad bags number turns to be 0 . The optimal objective value is $1.6207 \times 10^{6}$

\subsection{Polish-3012wp System}

Polish-3012wp system represents the Polish networks during winter 2007-08 evening peak, with 3012 buses, 502 generators and 3572 transmission line, which lead to 2510 nonlinear constraints in (2b). Thus, there are $3012 \times 3013 / 2=4537578$ complex scalar variables in the $W$ matrix. We apply the Large-Scale NOA as follow,

Initialization. Following (Madani et al., 2015a) to decompose the 3012 buses into 3012 overlapped bags. The largest bag size is 25 , while the smallest bag size is 1 . After the bags decomposition, the total number of the complex decision variables in (8) is 30996 vs 4537578 complex decision variables in (3). Solve (8) over the 3012 bags, we find that there are 381 bad bags, which means the rank of the 381 bags are more than 1.The largest bad bag size is 25 , while the smallest bad bag size is 2 . Other 2631 good bags are all rank-1. Then go to next step.

Step 1. Set $\varepsilon_{\text {tol }}=10^{-5}$ and put the 2631 good bags in constraint (9b), solve (9), after 5 iterations, the bad bags number turns to be 5 , while the good bags 
number turns to be 3007 . Among the 5 bad bags, two have size 3 , two have size 4 , and one has size 5 .

Step 2. Put the 3007 good bags in constraint (9b), solve (9), after 5 iterations, the bad bags number turns to be 0 . The optimal objective value is $2.6406 \times 10^{6}$.

\subsection{Polish-3120sp System}

Polish-3120sp system represents the Polish networks during summer 2008 morning peak, with 3120 buses, 505 generators and 3693 transmission line, which lead to 2615 nonlinear constraints in (2b). Thus, there are $3120 \times 3121 / 2=4868760$ complex scalar variables in the $W$ matrix. We apply the Large-Scale NOA as follow,

Initialization. Following (Madani et al., 2015a) to decompose the 3120 buses into 3120 overlapped bags. The largest bag size is 25 , while the smallest bag size is 1 . After the bags decomposition, the total number of the complex decision variables in (8) is 32637 vs 4868760 complex decision variables in (3). Solve (8) over the 3120 bags, we find that there are 21 bad bags, The largest bad bag size is 7 , while the smallest bad bag size is 2 . which means the rank of the 21 bags are more than 1. Other 3099 good bags are all rank-1. Then go to next step.

Step 1. Set $\varepsilon_{\text {tol }}=10^{-5}$ and put the 3099 good bags in constraint (9b), solve (9), after 5 iterations, only 2 bad bags with size 2 are still bad, while other bags turn good.

Step 2. Put the 3118 good bags in constraint (9b), solve (9), after 4 iterations, the bad bags number turns to be 0 . The optimal objective value is $2.1778 \times 10^{6}$.

It should be noted that, in step 2, the penalized term of bag- 81 was multiplied by 2 to increase the convergence speed.

\section{CONCLUSIONS}

OPF over power transmission networks is a difficult nonconvex optimization problem with numerous nonlinear equality and inequality constraints. We have developed a large-scale nonsmooth optimization algorithm to compute its optimal solution, which is efficient and practical for networks with reasonably large numbers of buses. Applications of NOA to OPF over three-phase power transmission networks are currently under consideration.

\section{REFERENCES}

Andersen, M. S., Hansson, A., and Vandenberghe, L. (2014). Reduced-complexity semidefinite relaxations of optimal power flow problems. IEEE Trans. Power Systems, 29(4):1855-1863.

Bai, X., Wei, H., Fujisawa, K., and Wang, Y. (2008). Semidefinite programming for optimal power flow problems. Elect. Power Energy Syst., 30(6-7):383392.

Bonnans, J. F., Gilbert, J. C., Lemarechal, C., and Sagastizabal, C. (2006). Numerical Optimization Theoretical and Practical Aspects (second edition). Springer.

Bukhsh, W., Grothey, A., McKinnon, K., and Trodden, P. (2013). Local solutions of the optimal power flow problem. IEEE Trans. Power Systems, 28(4):47804788.

Carpentier, J. (1962). Contribution to the economic dispatch problem. Bulletin Society Francaise Electriciens, 3(8):431-447.

Huneault, M. and Galiana, F. D. (1991). A survey of the optimal power flow literature. IEEE Trans. Power Systems, 6(2):762-770.

Lavaei, J. and Low, S. H. (2012). Zero duality gap in optimal power flow problem. IEEE Trans. Power Systems, 27(1):92-107.

Madani, R., Ashraphijuo, M., and Lavaei, J. (2015a). Promises of conic relaxation for contigencyconstrained optimal power flow problem. IEEE Trans. Power Systems, 30.

Madani, R., Sojoudi, S., and Lavaei, J. (2015b). Convex relaxation for optimal power flow problem: Mesh networks. IEEE Trans. Power Systems, 30:199-211.

Molzahn, D. K., Holzer, J. T., Lesieutre, B. C., and DeMarco, C. L. (2013). Implementation of a large-scale optimal power flow solver based on semidefinite programming. IEEE Trans. Power Systems, 28:39873998.

Momoh, J. A., El-Hawary, M. E., and Adapa, R. (1999). A review of selected optimal power flow literature to 1993. part I: Nonlinear and quadratic programming approaches. Solar Energy, 14(1):96-104.

Pandya, K. S. and Joshi, S. K. (2008). A survey of optimal power flow methods. J. of Theoretical and Applied Information Technology, 4(5):450-458.

Shi, Y., Tuan, H. D., Su, S., and Tam, H. H. M. (2015). Nonsmooth optimization for optimal power flow over transmission networks. In Proc. 2015 IEEE Global Conf. on Signal and Information Processing (GlobalSIP).

Zimmerman, R. D., Murillo-Sanchez, C. E., and Thomas, R. J. (2011). MATPOWER: steady-state operations, planning, and analysis tools for power systems research and education. IEEE Trans. Power Systems, 26(1):12-19. 